\title{
结合用户导向和遗传算法的医疗终端界面布局设计
}

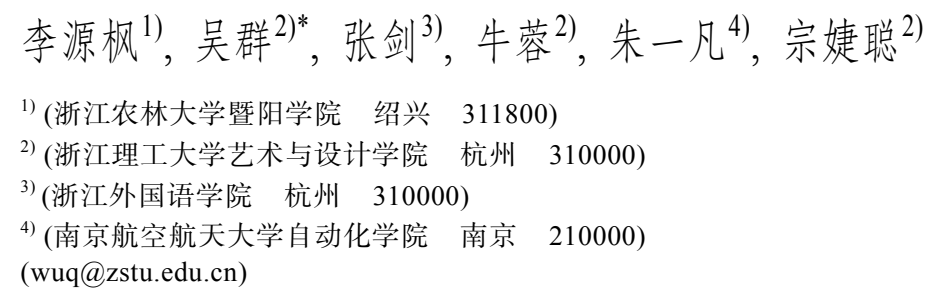

摘 要: 为了优化公共终端界面设计, 提升用户体验, 提出一种结合用户导向和遗传算法的界面布局方法. 该方法 将用户主观认知与客观视觉原则结合后转化为布局指标, 以界面布局方案的指标为自变量, 用户体验值为因变量, 将界面设计布局指标与主观用户体验之间的认知关系建模为可计算的函数. 在通过切片树方式对界面布局特征编码 后进行展开解空间搜索, 具体步骤包括生成树结构、确定切割方式获得最佳切片树和调整元素节点找到最佳布局. 以 医疗公共终端中挂号界面和交款界面为例, 使用该方法对其进行布局优化, 最后使用可用性测试进行验证. 实验结 果表明, 经过优化后的界面布局在各可用性指标上与初始界面的相比有明显的提升, 证明了该方法在医疗终端界面 设计场景的应用中具有有效性; 此外, 这类生成式辅助设计有助于减轻设计师的工作量, 提升工作效率.

关键词：医疗公共终端界面布局；用户导向；遗传算法；可用性测试；切片树

中图法分类号: TP391.41 DOI: 10.3724 SP.J.1089.2021.18338

\section{Layout Design of Medical Terminal Interface Combining User-Oriented and Genetic Algorithm}

\author{
Li Yuanfeng ${ }^{1)}$, Wu Qun ${ }^{2)^{*}}$, Zhang Jian ${ }^{3)}$, Niu Rong ${ }^{2)}$, Zhu Yifan ${ }^{4)}$, and Zong Jiecong ${ }^{2)}$ \\ 1) (Jiyang College of Zhejiang A\&F University, Shaoxing 311800) \\ 2) (School of Art and Design, Zhejiang Sci-Tech University, Hangzhou 310000) \\ 3) (Zhejiang International Studies University, Hangzhou 310000) \\ ${ }^{4)}$ (College of Automation Engineering, Nanjing University of Aeronautics and Astronautics, Nanjing 210000)
}

\begin{abstract}
In order to achieve the optimal interface design of the public terminal while improving user experience, this paper proposes an interface layout method that combines user orientation and a genetic algorithm. According to this method, the subjective cognition of users and their objective vision principle are integrated before conversion into a layout index. With the index of the interface layout scheme taken as the independent variable and the user experience value as the dependent variable, the cognitive relationship between the interface layout index and subjective user experience was modeled as a computable function. The expansion solution space was searched after the interface layout features were encoded using the slicing tree method. The specific steps include generating tree structure, determining the cutting method to obtain the
\end{abstract}

收稿日期: 2020-4-29; 修回日期: 2021-02-26. 基金项目: 国家自然科学基金(61972361); 教育部人文社科基金(18YJC760099); 杭 州市哲学社科规划项目(M21JC073). 李源枫(1990一), 男, 硕士, 讲师, 主要研究方向为用户体验设计、人机交互; 吴群(1978一), 男, 博士, 教授, 硕士生导师, CCF 会员, 论文通讯作者, 主要研究方向为工业设计、人机工程、可用性研究; 张剑(1978一), 男, 博士, 教 授, 主要研究方向为机器学习、多媒体信息处理、神经网络; 牛蓉(1994一), 女, 硕士研究生, 主要研究方向为可用性研究、工业设计; 朱一凡(1997一), 男, 硕士研究生, 主要研究方向为图像处理、计算机视觉; 宗婕聪(1992一), 女, 硕士研究生, 主要研究方向为产品 设计. 
best section tree, and adjusting the element nodes to identify the most satisfactory layout. With the registration interface and payment interface of the medical public terminal as an example, this method was applied to optimize its layout and then verified through a usability test. According to the experimental results, the usability indicators of the optimized interface layout was improved more significantly compared with the initial interface, suggesting the effectiveness of this method in the application of medical terminal interface design scenarios. Moreover, the generative auxiliary design of this kind is conducive to reducing the workload placed on designers and to enhancing efficiency.

Key words: medical terminal interface layout; user-oriented; genetic algorithm; usability test; slicing tree

许多用户在使用智能公共终端时发现诸多终 端界面的可用性较低，如用户在操作医疗类公共 终端界面时往往需要在服务人员的指导下完成. 这类现象在一定程度上是由于对公共终端界面布 局混乱, 操作烦琐等原因导致的. 这意味着智能公 共终端未能真实地根据用户需求进行界面设计. 因此, 如何基于用户导向对公共终端界面进行布 局设计, 已经成为提高界面操作可用性的重要手 段，也是当下的研究热点.

目前，针对用户界面的设计改良主要有 2 个方 向：一是从通过主观量表等方式搜集用户主观认 知数据改良用户界面, 以提高可用性; 二是从利用 算法或生成式设计等技术手段对界面展开布局设 计. 前者, Alves 等 ${ }^{[1]}$ 在设计前对用户的思维过程和 行为有关的某些心理因素进行了量化评估; Quinn 等 ${ }^{[2]}$ 通过测试来预测用户动作或纠正输人, 以辅助 界面设计、更广泛地对基于用户偏好的平台进行定 义. 上述研究很好地阐述了界面布局可用性的重 要性, 但分析过程只从用户认知角度展开, 研究结 果受被试者主观意愿影响较大. 后者有学者运用 算法对设施布局问题进行计算, 如 Tate 等 ${ }^{[3]}$ 成功地 使用数学编程技术建模并增强了约束条件; 文献 [4]运用两段式遗传算法对设备电路面积折中优化, 得到合理折中解. 这证明从客观技术角度解决布 局问题是可行的，但不足之处在于缺少基于用户 角度的主观认知的考虑.

针对公共终端界面布局问题，本文提出一种 结合用户导向与遗传算法的界面布局方法. 通过 建立界面设计布局指标与主观用户体验之间的映 射, 利用遗传算法模拟种群的进化过程, 生成界面 布局方案集. 并以医疗终端界面自助挂号界面和 付款界面布局设计为例，展开方案集的生成; 最后 选取 60 位用户，对生成的方案集和初始方案进行 可用性评估, 为后续界面设计提供依据.

\section{1 相关工作}

结合用户导向与遗传算法的界面布局方法主 要涉及用户体验设计规则和遗传算法的概念. 本 文将从这 2 个方面介绍相关工作.

用户体验设计主要的内容为用户界面，在行业 的发展过程中诞生了许多通用的设计准则. Sanders 等 ${ }^{[5]}$ 建立了界面元素排列的基本设计规则, 即要求 在操作顺序中使用的界面元素必须相对于这些顺序 进行定位, 要求元素的排布要符合用户的操作顺 序. 接近相容性规则 ${ }^{[6]}$ 要求同任务操作相关的元素 应相邻或靠近, 并且考虑界面中每个元素的使用 频率. 因此, 经常使用的元素必须占据便于快速操 作的位置, 高频元素所占据的位置和面积要方便 访问和使用. Cooper 等 ${ }^{[7]}$ 提出的极简规则要求目标 导向的交互设计反映用户的心智模型，消除界面 中不必要的复杂度. 但是, 类似关于用户体验的研 究通常在智能手机或是车载界面等个人设备上展 开, 而在公共终端界面设计方面的相关研究较少.

遗传算法 ${ }^{[8]}$ 是一种基于“优胜劣汰”的达尔文 生物进化论的并行、随机、自适应的优化算法. Diego-Mas 等 ${ }^{[9]}$ 使用遗传算法对界面布局进行优 化, 并成功地使用眼动仪和鼠标移动进行验证. 此 外，文献[10]将遗传算法运用到首饰设计中，基于 用户偏好生成了新的首饰方案. Srinivas 等 ${ }^{[11]}$ 的研 究证明了切片树和遗传算法的组合方法可用于优 化界面布局中控件和显示的布局, 对本文具有一 定的借鉴意义.

关于界面设计的研究非常多, 本文只介绍医 疗公共终端界面布局问题, 提出结合用户导向和 遗传算法的设计方法. 该方法在传统遗传算法的 基础上, 融合用户对医疗界面可用性问题的调研 结果, 并通过切片树的方法将主观认知问题转变 成计算指标, 通过计算获得最佳布局方案集. 


\section{2 本文方法}

结合通用布局规则和用户调研结果获得基于 用户导向的医疗布局原则，遗传算法从技术角度 对界面布局进行计算, 二者结合能更科学地解决 界面布局设计问题.

将搜集到的用户对医疗终端界面的主观认知 和文献中的设计规则相结合，建立基于用户导向 的布局原则. 使用切片树方法对界面布局原则和 方案进行转换, 形成相应布局指标. 以界面布局方 案的指标为自变量, 用户体验值为因变量, 建立适 应度函数. 在对布局特征进行编码后, 进人遗传算 法的计算流程中，解码后得到优化方案集. 最后, 通过对初始方案和生成方案进行可用性对比测试 得出结论. 图 1 所示为本文方法流程图.

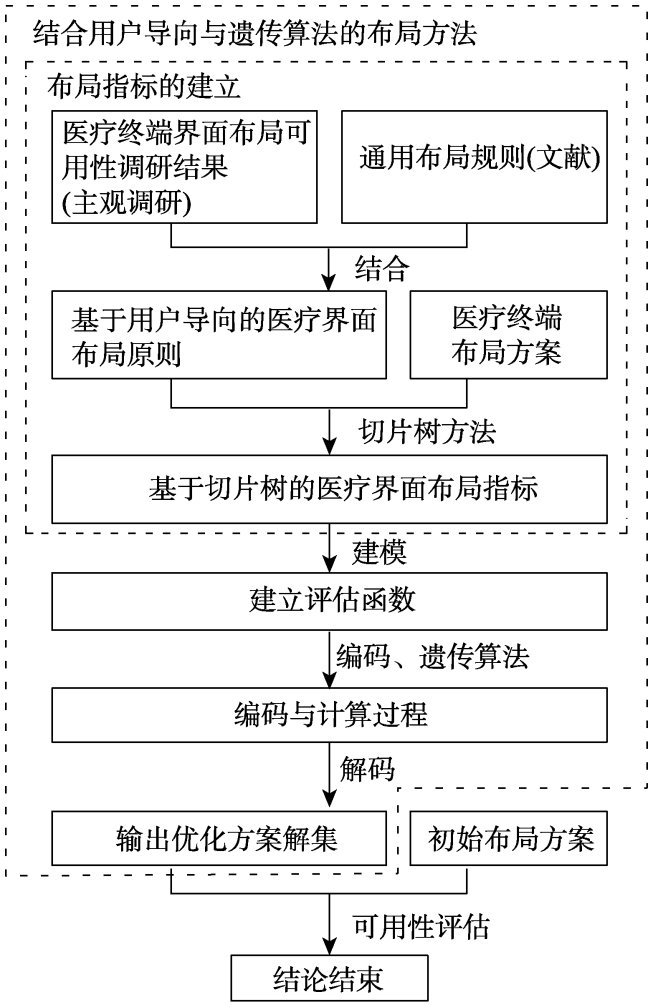

图 1 用户导向的界面布局优化流程

Step1. 制定基于用户导向的医疗布局设计原则. 根据用户主观调研结果结合通用布局原则制定医疗场 景下的界面布局原则。

Step2，通过切片树建立布局方案的计算模型，布 局原则的计算指标.

Step3. 建立适应度函数. 根据设计原则与编码方 式建立适应度函数

Step4. 布局特征编码. 将界面中的元素进行编码, 将设计规划问题转化成数学问题.
Step5. 遗传算法操作过程. 通过适应度函数计算 个体适应值, 并按照规则对种群执行遗传操作; 解码后 得到优化方案集.

Step6. 可用性测试. 优化方案集与初始方案进行 可用性对比试验.

\section{1 建立基于用户导向的医疗界面布局原则}

为了对当下医疗终端界面布局设计进行可用 性问题采集, 以现有用户认为可用性较差的浙江大 学医学院附属第二医院的医疗自助服务终端为调查 对象. 评估内容按照布局模块划分标头、导航、底 行栏、选择类型、确定与翻页键以及内容共 6 个模 块; 并根据模块搜集可用性评估问卷 (post-study system usability questionnaire, PSSUQ ${ }^{[12]}$, 通过线 上渠道下发问卷 315 份, 其中有效问卷 298 份. 最 终获得终端界面布局可用性问题如表 1 所示. 由于 这些可用性问题与文献[5-7]有一定相似性，因此 可使用通用设计规则解决表 1 中的问题, 对应关系 如表 2 所示.

表 1 医疗终端界面布局可用性问题

\begin{tabular}{ll}
\hline \multicolumn{1}{c}{ 模块 } & \multicolumn{1}{c}{ 可用性问题(来自调研结果) } \\
\hline 标头 & 图形展示面积占比过大, 无效信息过多 \\
导航 & 引导信息不明显, 无实质功能, 逻辑混乱 \\
底行栏 & 无效信息过多 \\
选择类型 & 操作步骤烦琐且混乱, 用户易误点, 需要减 \\
& 少步骤, 无关图标易误导用户 \\
确定与翻页键 & 医疗终端老年人使用较多, 按键不明显且与 \\
& 内容选择按键混淆, 对元素大小的适配要求 \\
内容 & 内容排列缺乏规律, 缺乏层次感, 内容类目 \\
& 过多, 寻找目的内容按键时间过长 \\
\hline
\end{tabular}

表 2 医疗终端界面布局可用性问题归类及 布局规则对应表

\begin{tabular}{|c|c|}
\hline 对可用性问题的归类(调研结果) & $\begin{array}{l}\text { 解决可用性问题的 } \\
\text { 布局规则(来自文献) }\end{array}$ \\
\hline 医疗终端老年人使用较多, 重要的、高 & 接近相容性规则 \\
\hline 频访问的功能按键面积比例需要调整 & \\
\hline 医疗类界面对有效性要求较高, 当下引 & 元素接 \\
\hline 导信息混乱, 易造成用户逻辑混乱 & \\
\hline 操作步骤烦琐, 同一操作任务下, 连续 & 极简规则 \\
\hline 按键不易寻找, 需要进行简化 & 接近相容性规则 \\
\hline 界面主体内容排列缺乏规律, 层次感较 & 接近相容性规则 \\
\hline 差, 寻找目标元素时间较长 & 元素排列顺序规则 \\
\hline
\end{tabular}

结合用户医疗界面主观体验反馈，以及界面 元素排列、接近相容性规则等通用布局设计规则, 提出基于用户导向的医疗布局设计原则如下：

原则 1. 操作流程频繁访问的界面元素在布局 中必须彼此靠近. 医疗服务终端中选择类型模块、 确定翻页模块、导航模块使用频率较高, 需要彼此 
靠近.

原则 2. 在操作同一任务时, 元素之间距离尽 量相互靠近. 在进行类型选择的任务中, 类型选择 按钮、点击内容按钮与确定翻页按钮作为前后点击 行为, 元素需要尽量相互靠近.

原则 3. 元素的面积及比例大小应根据其访 问和使用的频率确定. 医疗场景下，需要降低患 者对界面的认知成本. 因此, 类型选择模块(挂号 选择、科室选择模块) 、导航模块、确定与翻页模 块由于点击频率较高, 区域面积和按钮面积需要 适当放大.

原则 4. 在操作过程中, 可操作按键布局放置 在显著的区域. 在医疗终端界面中有内容模块的 区域尽量放置在界面的中心显著区域; 反之，仅作 为信息展示的非功能模块则放置在周边.

\section{2 建立数学模型及计算流程}

上述基于用户导向的医疗界面布局原则是整 合用户的主观需求和客观设计原则后建立的. 为 了将其量化并转化为可进行数学建模的指标, 引 人切片树的方法对布局问题进行编码 ${ }^{[13]}$, 将布局 原则转变为切片树相应的计算指标几何势 $p_{\mathrm{G}}$ 和相 关势 $p_{R}$. 界面中的元素除了需要遵循几何限制, 还必须保持交互成本最小的方式来获得平面区域 上元素的布局 ${ }^{[14]}$, 在本文中用户所消耗的交互成 本越低，该方案的用户体验值 $E_{\mathrm{f}}$ 就越好.

因此，在数学建模的过程中，整个模型的输人 $X$ 为基于各布局原则的布局方案所对应的计算指标 $\left(p_{\mathrm{G}}\right.$ 和 $\left.p_{\mathrm{R}}\right)$; 输出 $Y$ 为用户体验值 $E_{\mathrm{f}}$. 最后, 通过 遗传算法求得整个函数为求 $E_{\mathrm{f}}$ 极小值所对应的布 局指标 $\left(p_{\mathrm{G}}\right.$ 和 $\left.p_{\mathrm{R}}\right)$ 的值, 最终解码得到优化后各元 素布局方案集. 该部分的流程如图 2 所示.

\subsection{1 建立基于切片树的布局指标体系}

\subsubsection{1 建立布局方案的数学模型}

为解决布局问题，采用两阶段遗传算法 ${ }^{[14-15]}$, 每个布局方案都可以转换为对应的切片树. 图 3 所 示为医疗挂号机初始界面对应分割结构和对应切 片树，该结构由 6 个不同形状的非重叠分区组成, 也称为元素; 图中带有数字编号的圆为元素, 也称 为叶子, 切割节点用方块表示; 切割方式为水平切 割 $h$ 或垂直切割 $v$, 而切割比例控制着元素的长宽 比. 通过修改内部节点中进行切割的类型，可以生 成不同的布局. 生成一个切片树的基本步骤如下:

Step1. 生成树的结构(页面结构).

Step2. 确定切割方式，获得最佳切片树后(决定切

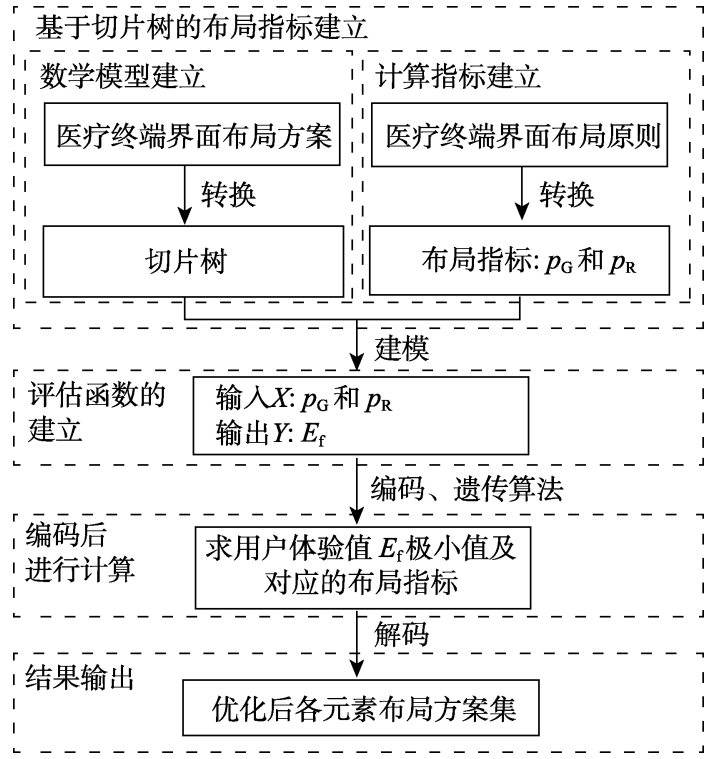

图 2 建立数学模型及计算流程

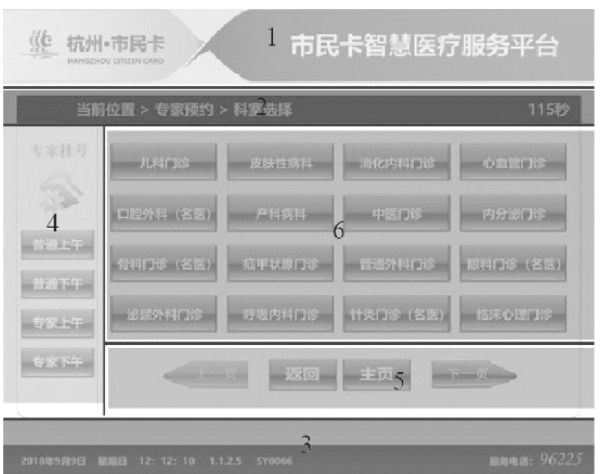

a. 初始界面元素分割

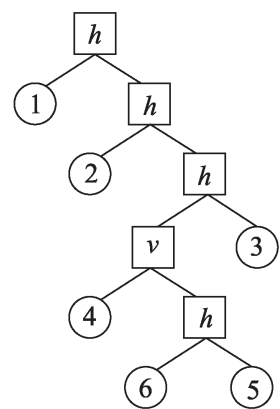

b. 初始界面对应切片树

图 3 医疗挂号机初始界面结构布局与对应的切片树

割方式即横切或纵切).

Step3. 调整元素数值(节点微调), 即调整各元素切 割比例，最终找到最佳布局.

\subsubsection{2 建立布局原则的计算指标}

除布局方案本身与切片树的转换外, 由于各 布局元素是有取值范围的，即布局的几何限制 ${ }^{[14]}$, 同时又要满足基于用户导向的医疗布局界面原则. 因此引人切片树的几何势 $p_{\mathrm{G}}$ 和相关势 $p_{\mathrm{R}}$ 概念, 
可通过切片树完成对布局界面原则的指标转换与 计算.

(1) 相关势 $p_{R}$

采用相关势 $p_{\mathrm{R}}$ 指标用于测量元素间的相邻程 度以及用户操作时的便捷程度. 其在物理上表现 为当执行同一任务时, 要求几个相关按键相邻，同 时用户在尽量少的步骤内完成操作. 具体方法是 将用户行为路径步骤数转换为切片树结构元素之 间的步长. 使得各元素两两之间相邻概率与步长 倒数的乘积之和的值, 即切片树结构中元素之间 的步长最小化, 各元素两两之间相邻的概率最大 化. 相关势的功能影响各元素排列, 因此原则 1 和 原则 2 中基于界面元素间的顺序和距离均与其相 关. $p_{\mathrm{R}}$ 的表达式为

$$
p_{\mathrm{R}}=\sum_{i=1}^{n} \sum_{j=1}^{n}(P(i j) \cdot f(i j))
$$

其中, $f(i j)$ 表示在切片树结构上元素 $i$ 到 $j$ 的步 长的倒数; $P(i j)$ 表示元素 $i$ 和 $j$ 的相邻概率; $n$ 表 示元素数量. 当元素之间相邻概率 $P(i j)$ 越大, 步 长 $1 / f(i j)$ 越小, 那么 $p_{\mathrm{R}}$ 值越大.

(2) 几何势 $p_{\mathrm{G}}$

采用几何势指标评估一个切片树生产其元素 符合几何限制的布局能力 ${ }^{[14]}$. 其在物理上表现为 当限制越低时, 切割点滑动范围越大, 获得优化切 割的可能性也越高. 具体表现为扩大布局区域, 减 少约束条件, 如增加元素高宽比的范围.

由于几何势所表现的是对布局元素的几何限 制，这与上文中界面布局原则 3 形成对应，所相关 的阶段为切割比例调整阶段. 原则 4 中将可操作按 键布局放置在显著区域的限制要求，也与几何势 对应. 由于原则 4 必须在切割一开始就展开，且影 响切片树的生成, 因此它所处阶段在改变树的结 构、切割方式 2 个阶段. 当对界面进行最优化的切 割时，提供较低不合规性的切片树的几何势将较 低，其表达式为

$$
p_{\mathrm{G}}=\min \left(U_{\mathrm{h}}(\partial), U_{\mathrm{v}}(\partial)\right)
$$

其中， $p_{\mathrm{G}}$ 是基本树的几何势; $\partial$ 是初始域比率; $U_{\mathrm{h}}$ 和 $U_{\mathrm{v}}$ 是分别对初始域进行水平和垂直切割的 情况下几何限制的不符合的总和. 当 $U_{\mathrm{h}}$ 和 $U_{\mathrm{v}}$ 的 值越小时, $p_{\mathrm{G}}$ 的值越小.

几何势和相关势能有效地表达切片树的结构、 切割方式和切割比例，也解决了界面布局的几何限 制问题. 因此，基于用户导向的布局原则与切片树 各个阶段关联及计算指标的对应关系如表 3 所示.
表 3 基于用户导向的布局原则与切片树指标的对应

\begin{tabular}{clc}
\hline 原则 & \multicolumn{1}{c}{ 切片树生成所属阶段 } & 切片树的计算 \\
\hline 1 & 改变树的结构 & $p_{\mathrm{R}}$ \\
2 & 改变树的结构 & $p_{\mathrm{R}}$ \\
3 & 切割比例 & $p_{\mathrm{G}}$ \\
4 & 改变树的结构、切割方式 & $p_{\mathrm{G}}$ \\
\hline
\end{tabular}

综上所述，基于用户导向的医疗界面布局原 则以及布局方案本身可通过切片树工具从认知问 题转化为数学建模问题.

\subsection{2 建立评估函数}

在完成将用户导向的布局原则转换成切片树 指标后，通过建立评估函数满足医疗终端界面布 局各元素之间的交互关系消耗值，以保持在一定 水平 ${ }^{[14]}$. 基于切片树的几何势 $p_{\mathrm{G}}$ 和相关势 $p_{\mathrm{R}}$ 构 建评估函数 $E_{\mathrm{f}}\left(p_{\mathrm{G}}, p_{\mathrm{R}}\right), E_{\mathrm{f}}$ 表示用户操作界面时 的消耗值即用户体验值.

用户体验值 $E_{\mathrm{f}}$ 是界面布局方案指标 $p_{\mathrm{R}}$ 和 $p_{\mathrm{G}}$ 之间的映射，整个函数为求 $E_{\mathrm{f}}$ 极小值时所对应的 $p_{\mathrm{R}}$ 和 $p_{\mathrm{G}}$, 解码后得到元素布局方案集. 当 $p_{\mathrm{G}}$ 越 大， $p_{\mathrm{R}}$ 值越小时，才能保证用户体验值 $E_{\mathrm{f}}$ 越小. 当用户执行任务时，用户路径越短或者所消耗时 间越少, 则消耗值越低, 用户体验越好. 评估函数 $E_{\mathrm{f}}\left(p_{\mathrm{G}}, p_{\mathrm{R}}\right)$ 表达式为

$$
E_{\mathrm{f}}\left(p_{\mathrm{G}}, p_{\mathrm{R}}\right)=c_{\mathrm{G}} \cdot p_{\mathrm{G}}+c_{\mathrm{R}} \cdot \frac{1}{1+p_{\mathrm{R}}}
$$

其中， $E_{\mathrm{f}}$ 为用户体验值 (因变量), $p_{\mathrm{G}}$ 和 $p_{\mathrm{R}}$ 为界面 布局方案指标(自变量); $c_{\mathrm{G}}$ 和 $c_{\mathrm{R}}$ 分别是几何准则 和关系准则之间的相对重要性的加权系数. 其中, 分母中的加 1 是为了防止整个分母为 0 .

\subsection{3 编码与计算过程}

根据切片树对医疗界面布局进行编码，随后 进人遗传算法流程. 界面中每个元素编码代表一 个基因，一个界面布局方案为一个个体，多个界面 布局方案组成一个种群. 与第 2.2.1 节切片树形成 过程相同，编码的方法也是将布局解构为 3 个部分 的字符串, 分别表示树结构编码、切割方式编码和 切割比编码. 首先, 对切片树的树结构进行编码, 树结构编码共有 $n_{\text {Block }}+n_{\text {Device }}$ 个位置, 即元素(叶 子) 的数量 $n_{\text {Block }}$ 加上节点 (非叶子节点) 的数量 $n_{\text {Device, }}$ 如在元素的位置编码为 1 , 在节点的位置 编码为 0 ; 切割方式编码信息表示每一次切割是横 切或纵切, 如将横切 $h$ 编码为 0 或纵切 $v$ 编码为 1 , 切割方式的编码位数由节点数 $n_{\text {Device }}$ 决定; 根据 
上述 2 步可获得完整的树，而切割比决定了元素的 面积大小和形状, 其编码为每个元素的长宽比值, 即每个元素的 $\partial$ 值, 编码位数为元素的数量 $n_{\text {Block }}$. 最终, 将整个布局方案转换成了字符串, 完成编 码，如图 4 所示.

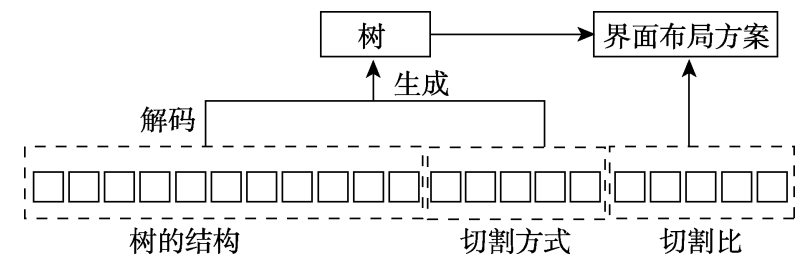

图 4 方案编码组成示意图

在完成了编码后进人计算过程. 在遗传算法 优化流程中，每一组编码都对应一个布局方案，使 用目标函数评估每个方案的用户体验值 $E_{\mathrm{f}}$. 操作 目标为淘汰元素间产生的较大消耗值的个体，而 消耗值较低的个体进人下一代进化流程中. 当函 数收玫后得到最小消耗值的种群，再对其映射的 布局指标解码, 以获得优化方案. 遗传算法的算法 流程如图 5 所示.

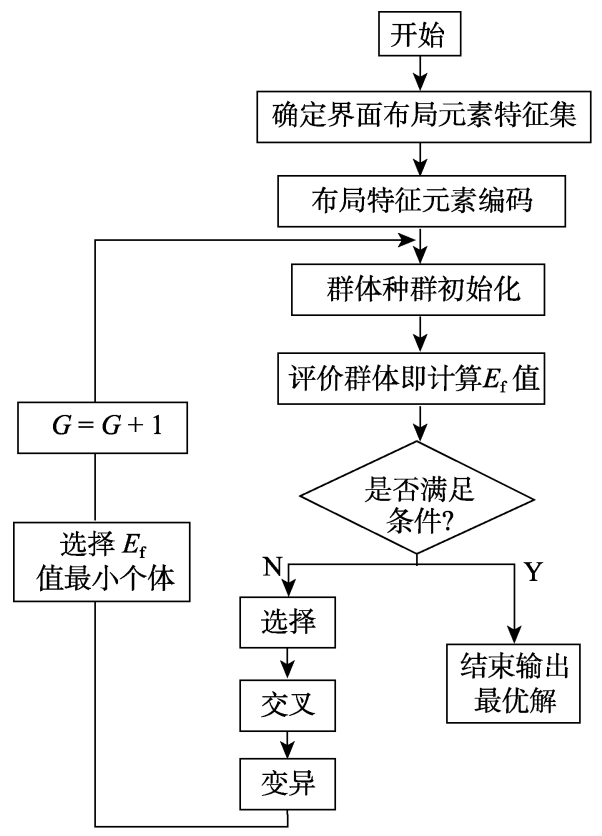

图 5 遗传算法流程

\section{3 实 验}

\section{1 界面布局的生成}

\section{1 .1 选取实验对象}

以浙江大学第二附属医院的医疗自助终端作 为研究载体. 由第 2.1 节可知, 在对所搜集的关于
医疗终端问卷整理汇总后，发现出现可用性问题 最多的是科室选择界面, 故选取挂号界面进行布 局优化. 如图 6 所示, 科室选择界面(高 $40.5 \mathrm{~cm}$, 宽 $51 \mathrm{~cm}$ )分成 6 类模块元素. 本文只对界面布局进 行研究, 不考虑色彩的影响, 并且将其还原成高保 真原型.
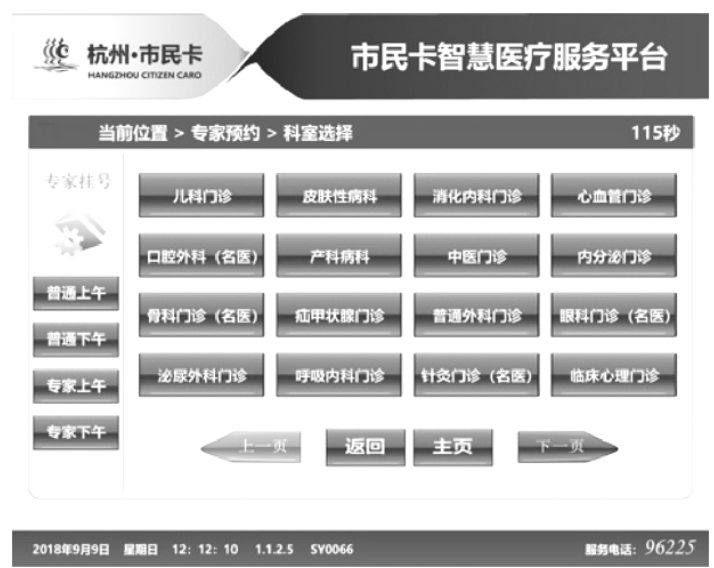

a. 初始界面

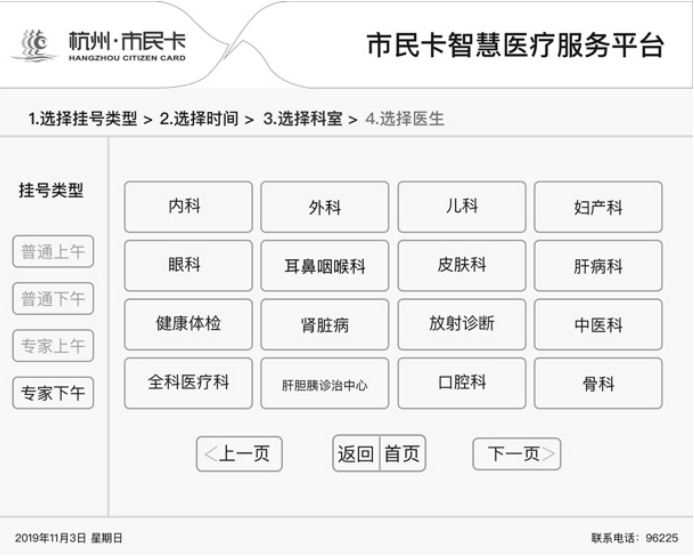

b. 初始界面的高保真原型

图 6 初始界面及其高保真原型

\subsection{2 参数设置}

算法的界面优化配置环境是在 Jupyter-Notebook 6.03 中进行, 由 Python 语言编写实现界面优 化. 界面布局优化的初始种群根据界面特征元素 随机产生 8 个, 采用轮盘奢法选择、交叉和变异; 交叉率和变异率均为 0.3 , 点击调试对其进行自动 排布, 以形成最优化布局, 程序迭代代数设置为 2000 代.

\section{2 可用性对比测试}

生成优化界面后, 需要通过可用性测试对初 始界面布局与生成的界面布局集进行可用性对比, 以验证该方法的有效性.

实验要求被试意识清楚, 能与调查员进行有 
效沟通, 共选取 60 名被试. 为了排除年龄和性别 因素的影响，其中，年龄大于等于 50 岁的老年人 30 人; 20 50 岁的中青年 30 人; 男性人数为 32 人, 女性 28 人, 被试人数分布如表 4 所示. 在实验开始 前, 研究人员给被试讲解当前界面的操作任务及 各元素的功能, 在确保每位被试明确任务流程及 功能后进人操作过程. 在选定测试任务时, 主要考 虑各功能的使用频率和普遍性，选择了较为常见 的眼科与较为不常见的中药门诊挂号任务, 以及 最常用的回到主页功能，操作任务如表 5 所示.

表 4 被试人数分布

\begin{tabular}{ccc}
\hline 性别 & 老年人 & 中青年人 \\
\hline 男 & 14 & 18 \\
女 & 16 & 12 \\
\hline
\end{tabular}

表 5 被试操作任务

\begin{tabular}{cl}
\hline 编号 & \multicolumn{1}{c}{ 任务 } \\
\hline 1 & 完成一个上午眼科门诊的专家号 \\
2 & 完成一个下午中药门诊的普通号 \\
3 & 回到主页 \\
\hline
\end{tabular}

可用性评估标准使用的是国际通用指标 ${ }^{[16]}$, 统计被试完成任务率(有用性), 每个被试的测试绩 效即完成任务时间(有效性), 系统满意度. 其中，系 统满意度量表(system usability scale, SUS) ${ }^{[16]}$ 通过 10 个与满意度相关的结构性问题进行分数选择, 每题选项为 5 分制的 $0 \sim 4$ 分 (0 分为非常不满意, 4 分为非常满意, 共 40 分). 被试完成任务后, 对每 道题所得分值相加后转变为百分制, 以获得最终 SUS 分值.

\section{4 实验结果}

\section{1 布局方案的生成结果}

当评估函数 $E_{\mathrm{f}}\left(p_{\mathrm{G}}, p_{\mathrm{R}}\right)$ 求得值为最小值时, 程序输出理论最优解和特征元素布局. 如图 7 所 示, 纵坐标代表用户体验值 $E_{\mathrm{f}}$, 横坐标代表切片 树遗传算法的迭代次数; 从图 7 中可得在迭代到 709 代时曲线波动开始减缓; 在迭代到 1411 代时 曲线开始趋于收玫, 得到最优解为 -4.1801719 . 根据适应度值随机共生成的 8 个科室界面布局优 化解, 其中的数字为不同模块的标号, 方案如图 8 所示.

\section{2 可用性测试对比结果与分析}

由于单独将生成布局方案集进行可用性对比

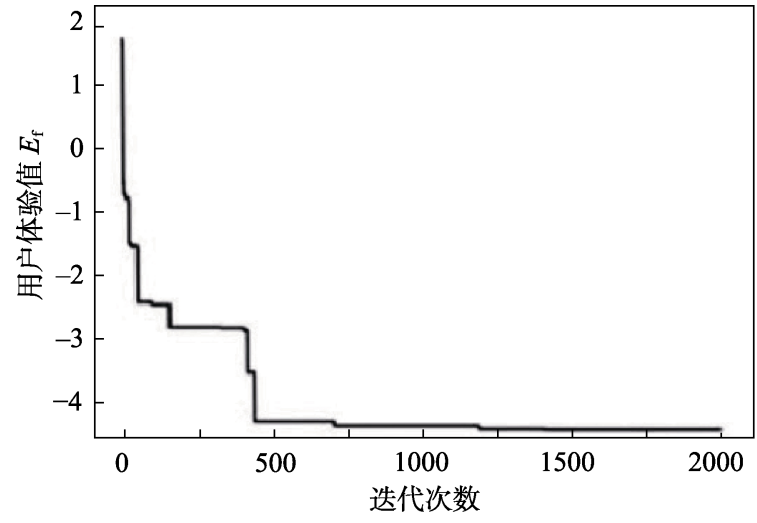

图 7 科室界面布局得到方案的收敛过程

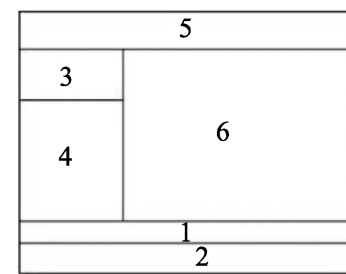

a. 方案 1

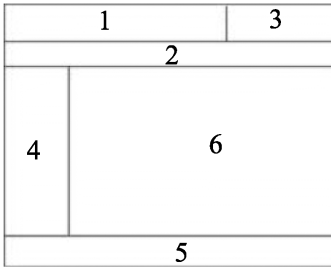

c. 方案 3

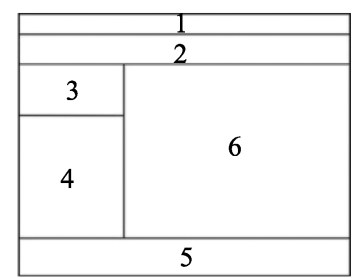

e. 方案 5

\begin{tabular}{|l|l|l|}
\hline 4 & 1 & 3 \\
\hline 2 \\
\hline \\
\hline 3 \\
\hline \\
\hline \multicolumn{3}{|c|}{} \\
\hline
\end{tabular}

g. 方案 7

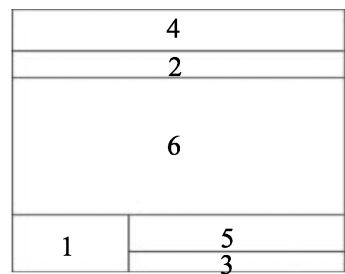

b. 方案 2

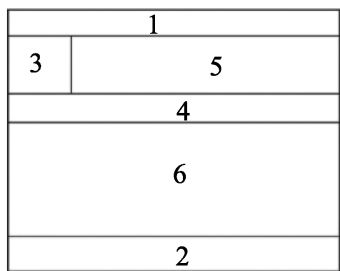

d. 方案 4

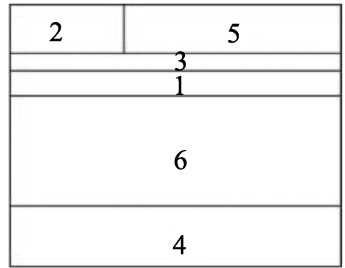

f. 方案 6

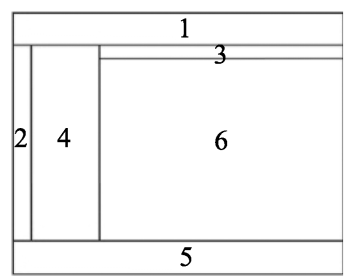

h. 方案 8
图 8 科室界面优化布局方案

没有意义, 且为了避免除布局因素外其他因素干 扰，因此使用高保真原型作为实验载体进行可用 性对比测试. 对比实验的载体为方案 1 方案 8 的 高保真原型和初始界面, 如图 9 所示.

表 6 中的统计结果表明, 在有用性方面, 除了 初始界面(96.9\%)、方案 4(87.1\%)、方案 5(80.4\%) 


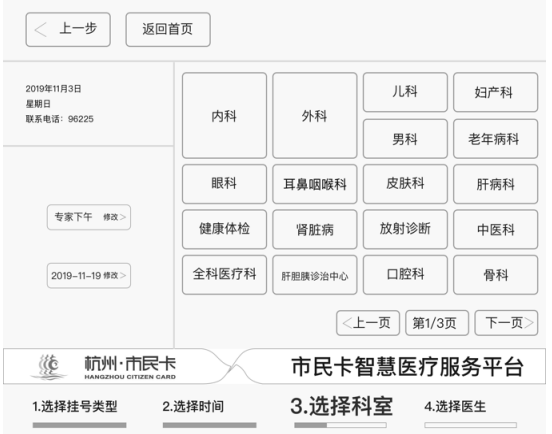

a. 方案 1

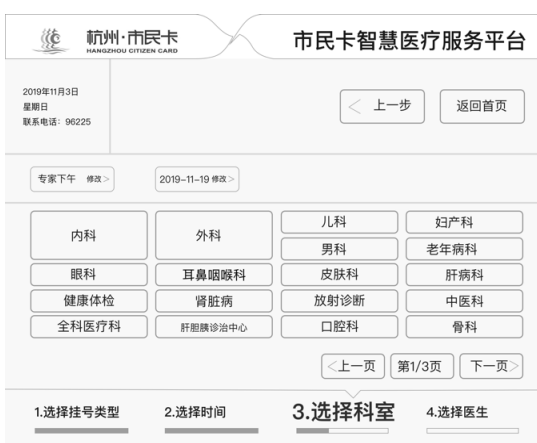

d. 方案 4

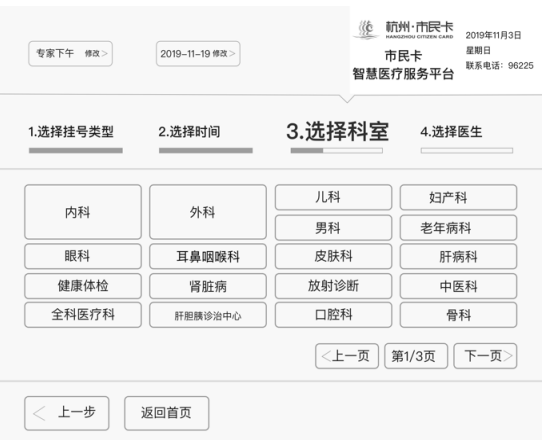

g. 方案 7

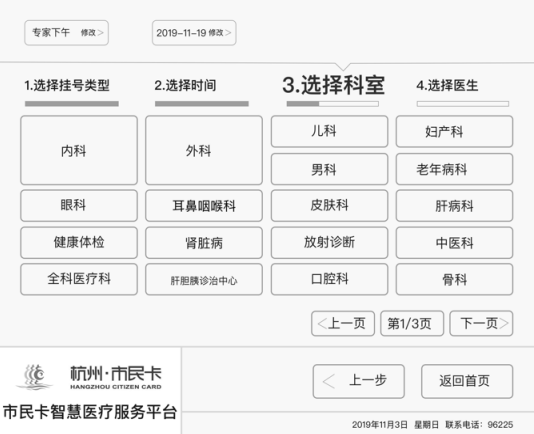

b. 方案 2

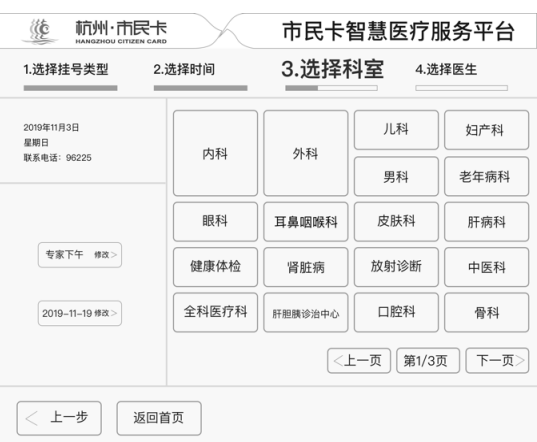

e. 方案 5

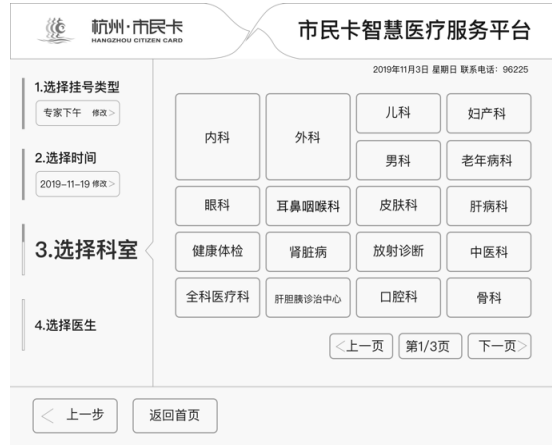

h. 方案 8

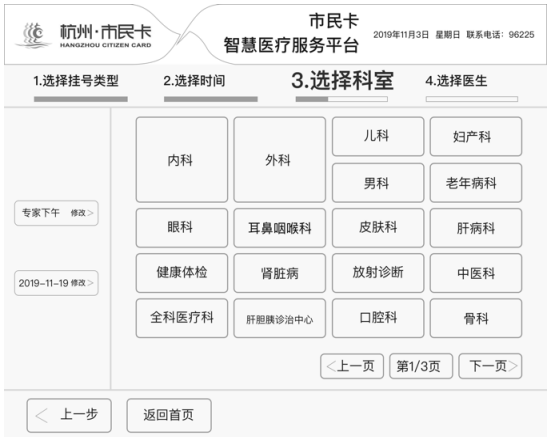

c. 方案 3

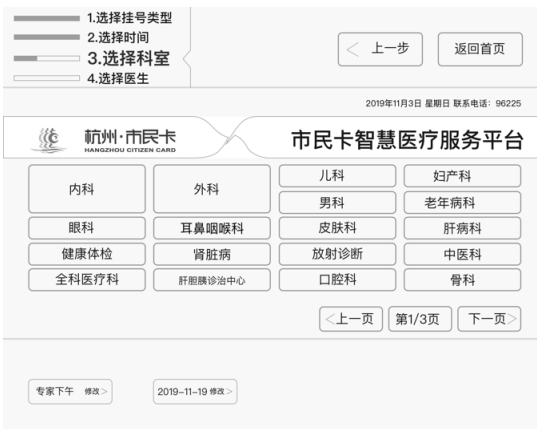

f. 方案 6

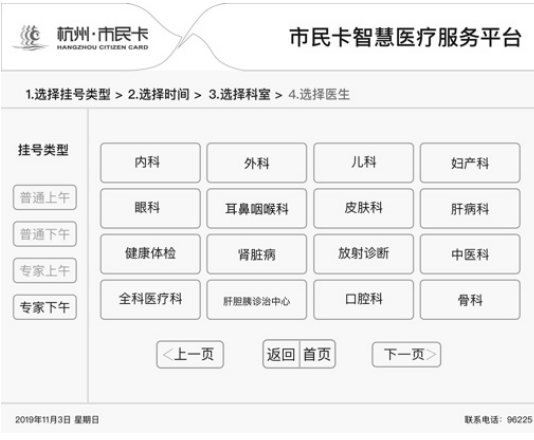

i. 初始方案

图 9 各方案及初始界面的高保真原型

外, 被试均完成了操作任务, 其余所有方案有用性 均为 $100 \%$; 在有效性方面, 使用时间进行绩效统 计, 单位为秒, 除了方案 4(9.12 s) 和方案 $5(8.91 \mathrm{~s})$ 外, 其余通过计算机生成的方案绩效均优于初始 方案, 原因可能是被试不习惯将确定与翻页模块 放置在上方, 导致绩效测试结果不理想; 在满意度 方面, 除了方案 4(66.1)、方案 5(69.2)外, 其余方案 均高于初始方案. 方案 8 的数据 $(100 \%, 4.99 \mathrm{~s}$, 84.4)在 9 个方案中最优.

此外，为了分析年龄因素对测试结果的影响， 研究人员对不同年龄段用户的平均绩效进行对比 分析. 表 7 的统计结果表明, 优化后的方案对老年 人的影响较大，即有效性提升较快; 而中青年人的
测试绩效略有提升. 这可能是由于中青年人已经 习惯使用电子产品, 对界面设计的容错率较高, 而 通过优化改良后的整体布局对老年人更加友好, 因此绩效有大幅提升. 在显著性方面, $P=0.0071$, 小 于 0.05 , 说明年龄因素对布局方案的有效性(绩效) 具有显著差异. 在绩效表现方面, 方案 8 在分年龄 段和整体的测试条件下绩效均是最优的, 因此方 案 8 为最优布局, 如图 10 所示. 此外, 本文还使用 该方法对医疗终端的付款界面进行优化, 其初始界 面优化后同样可以获得可用性良好的界面布局.

综上所述, 本文提出的结合用户导向和遗传 算法对初始方案进行优化布局、生成的界面布局可 用性表现良好，证明了该方法有效性. 
表 6 各方案可用性指标对比

\begin{tabular}{cccc}
\hline 方案 & 有用性 $/ \%$ & 有效性(绩效)/s & SUS 满意度 \\
\hline 初始 & 96.9 & 7.35 & 74.6 \\
1 & 100.0 & 6.31 & 75.9 \\
2 & 100.0 & 7.23 & 75.2 \\
3 & 100.0 & 6.67 & 84.2 \\
4 & 87.1 & 9.12 & 66.1 \\
5 & 80.4 & 8.91 & 69.2 \\
6 & 100.0 & 5.33 & 82.4 \\
7 & 100.0 & 6.67 & 77.3 \\
8 & 100.0 & 4.99 & 84.4 \\
\hline
\end{tabular}

表 7 年龄因素对方案有效性的影响分析

\begin{tabular}{cccc}
\hline \multirow{2}{*}{ 方案 } & \multicolumn{2}{c}{ 有效性(绩效)/s } & \multirow{2}{*}{$P$} \\
\cline { 2 - 3 } & 老年人 & 中青年人 & \\
\hline 初始 & 8.56 & 6.14 & \\
1 & 7.50 & 5.12 & \\
2 & 8.33 & 6.13 & \\
3 & 7.98 & 5.36 & \\
4 & 11.26 & 6.98 & 0.0071 \\
5 & 11.00 & 6.80 & \\
6 & 5.87 & 4.79 & \\
7 & 6.67 & 6.25 & \\
8 & 5.42 & 4.56 & \\
\hline
\end{tabular}

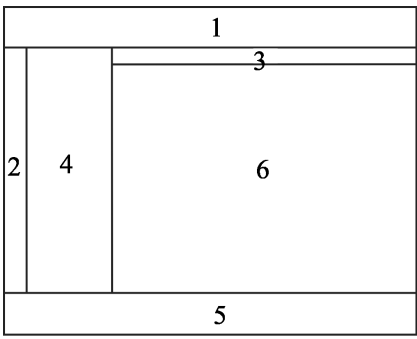

a. 方案 8

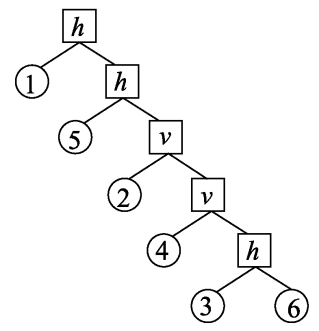

b. 方案 8 对应的切片树
图 10 最佳优化布局方案

\section{5 结 语}

本文提出结合用户导向和遗传算法界面布局 方法，将界面设计布局指标与主观用户体验之间 的认知关系建模为可计算的函数，通过计算流程 自动生成优化布局. 在对初始布局方案和生成布 局方案进行可用性对比测试后得出以下结论:

（1）使用结合用户导向和遗传算法的布局方法 所生成的布局方案在可用性指标上优于初始界面.

（2）切片树结合遗传算法的方法在医疗场景下 能给出高可用性的界面方案，证明该方法的有效.

(3) 该类计算机辅助生成式设计方法可以作 为设计辅助工具提升设计效率.

（4）医疗场景下，界面布局的优化对老年人影 响较大, 各项可用性指标有明显提升.

\section{参考文献(References):}

[1] Alves T, Natálio J, Henriques-Calado J, et al. Incorporating personality in user interface design: a review[J]. Personality and Individual Differences, 2019, 155: 109709

[2] Quinn P, Cockburn A. Loss aversion and preferences in interaction[J]. Human-Computer Interaction, 2020, 35(2): 143-190

[3] Tate D M, Smith A E. Unequal-area facility layout by genetic search[J]. IIE Transactions, 1995, 27(4): 465-472

[4] Bu Dengli, Jiang Jianhui, Luo Wenlang. Two-phase GA based area and SER trade-off algorithm for MPRM circuits[J]. Journal of Computer-Aided Design \& Computer Graphics, 2017, 29(10): 1924-1934(in Chinese)

（下登立，江建慧，罗文浪. 基于 2 个阶段遗传算法的 MPRM 电路面积与 SER 折中优化[J]. 计算机辅助设计与图形学学 报, 2017, 29(10): 1924-1934)

[5] Sanders M S, McCormick E J. Human factors in engineering and design[M]. 6th ed. New York: Tata McGraw-Hill, 1982

[6] Wickens C D, Carswell C M. The proximity compatibility principle: its psychological foundation and relevance to display design[J]. Human Factors: the Journal of the Human Factors and Ergonomics Society, 1995, 37(3): 473-494

[7] Cooper A, Reimann R, Cronin D, et al. About Face 4: the essentials of interaction design[M]. 4th ed. Beijing: Publishing House of Electronics Industry, 2015: 18-24(in Chinese)

(Cooper A, Reimann R, Cronin D, et al. About Face 4: 交互设 计精髓 $[\mathrm{M}]$. 倪卫国, 刘松涛, 薛菲, 等译. 4 版. 北京: 电子 工业出版社, 2015: 18-24)

[8] Goldberg D E, Holland J H. Genetic algorithms and machine learning[J]. Machine Learning, 1988(3): 95-99

[9] Diego-Mas J A, Garzon-Leal D, Poveda-Bautista R, et al. User-interfaces layout optimization using eye-tracking, mouse movements and genetic algorithms[J]. Applied Ergonomics, 2019, 78: 197-209

[10] Zhang Kaiwen, Shi Jian. Research on jewelry design based on genetic algorithms[J]. Machine Design \& Research, 2019, 35(5): 108-111+125(in Chinese)

(张凯文, 施健. 基于遗传算法的首饰设计研究 $[\mathrm{J}]$. 机械设 计与研究, 2019, 35(5): 108-111+125)

[11] Srinivas M, Patnaik L M. Adaptive probabilities of crossover and mutation in genetic algorithms[J]. IEEE Transactions on Systems, Man, and Cybernetics, 1994, 24(4): 656-667

[12] Lewis J R. Psychometric evaluation of the post-study system usability questionnaire: the PSSUQ[C] //Proceedings of the Human Factors \& Ergonomics Society Annual Meeting. Atlanta: Proceedings of the Human Factors Society, 1992: 36: 1259-1260

[13] Klausnitzer A, Lasch R. Optimal facility layout and material handling network design[J]. Computers \& Operations Research, 2019, 103: 237-251

[14] Diego-Mas J A, Santamarina-Siurana M C, Alcaide-Marzal J, et al. Solving facility layout problems with strict geometric constraints using a two-phase genetic algorithm[J]. International Journal of Production Research, 2009, 47(6): 1679-1693

[15] Erfani B, Ebrahimnejad S, Moosavi A. An integrated dynamic facility layout and job shop scheduling problem: a hybrid NSGA-II and local search algorithm[J]. Journal of Industrial and Management Optimization, 2018, 16(4): 1801-1834

[16] Nielsen J. Usability inspection methods[C] //Proceedings of Conference Companion on Human Factors in Computing Systems. New York: Association for Computing Machinery Press, 1994: 413-414 